\title{
Upregulated HSPA2 predicts early relapse of pancreatic cancer after surgery
}

\author{
Lu-Lu Zhai ${ }^{1 \wedge}$, Pei-Pei Qiao ${ }^{2}$, Yue-Shen Sun ${ }^{3}$, Zhi-Gang Tang ${ }^{1 \wedge}$, Tong-Fa Ju $^{4}$ \\ ${ }^{1}$ Department of General Surgery, Renmin Hospital of Wuhan University, Wuhan, China; ${ }^{2}$ Ningxia Medical University, Yinchuan, China; ${ }^{3}$ Peking \\ Union Medical College Hospital, Chinese Academy of Medical Sciences and Peking Union Medical College, Beijing, China; ${ }^{4}$ Department of \\ General Surgery, Affiliated Hangzhou First People's Hospital, Zhejiang University School of Medicine, Hangzhou, China \\ Contributions: (I) Conception and design: LL Zhai, ZG Tang, TF Ju; (II) Administrative support: ZG Tang, TF Ju; (III) Provision of study materials \\ or patients: LL Zhai, PP Qiao, YS Sun; (IV) Collection and assembly of data: LL Zhai, ZG Tang, TF Ju; (V) Data analysis and interpretation: LL \\ Zhai, PP Qiao, YS Sun; (VI) Manuscript writing: All authors; (VII) Final approval of manuscript: All authors. \\ Correspondence to: Prof. Zhi-Gang Tang. Department of General Surgery, Renmin Hospital of Wuhan University, 99 Zhangzhidong Road, Wuhan \\ 430060, China. Email: tzg567@163.com; Dr. Tong-Fa Ju. Department of General Surgery, Affiliated Hangzhou First People’s Hospital, Zhejiang \\ University School of Medicine, Hangzhou 310006, China. Email: jeffer_t@163.com.
}

Background Heat shock protein A2 (HSPA2) is known to relate to the pathogenesis and progress of cancer. This study aimed to investigate the connection between HSPA2 and early postsurgical relapse of pancreatic cancer (PC).

Methods: Expression of HSPA2 in 85 pairs of cancerous and matched noncancerous samples was determined by immunostaining method. The relationship between HSPA2 expression and early postsurgical recurrence was assessed using logistic regression. The performance and potential application of HSPA2 expression to predict early postsurgical recurrence was evaluated by receiver operating characteristic (ROC) curve analysis and decision curve analysis (DCA).

Results: HSPA2 expression in tumor specimens was markedly elevated compared with non-tumor specimens. Logistic regression analysis indicated that HSPA2 upregulation was an independent risk marker for early postsurgical recurrence of PC. ROC curve analysis and DCA demonstrated that both the area under the curve (AUC) and the net benefit of HSPA2 expression were higher than those of other clinicopathologic features in predicting early postsurgical relapse of PC. The combination of HSPA2 expression with other malignant clinicopathologic characteristics had greater AUC and net benefit relative to them alone in predicting early postsurgical recurrence.

Conclusions: Upregulated HSPA2 independently predicts early postsurgical recurrence of PC and has superior predictive performance and potential application value when combined with malignant clinicopathologic features. Our findings reveal that HSPA2 is a promising predictor for early postoperative relapse of $\mathrm{PC}$.

Keywords! Heat shock protein A2 (HSPA2); pancreatic cancer (PC); surgery; recurrence; risk factor

Submitted Apr 20, 2021. Accepted for publication May 26, 2021.

doi: $10.21037 /$ gs-21-262

View this article at: https://dx.doi.org/10.21037/gs-21-262

\footnotetext{
^ ORCID: Lu-Lu Zhai 0000-0003-0839-7316; Zhi-Gang Tang 0000-0001-5390-9670.
} 


\section{Introduction}

Pancreatic cancer (PC) is an extremely invasive and quickly progressive human digestive system malignant tumor, which is the seventh main reason for cancer-associated deaths globally (1). Statistic data from the American Cancer Society indicate that the disease has jumped to the fourth main reason for malignancy-associated deaths domestically, with a five-year relative survival rate of approximately $9 \%$ for all stages and races from 2009 to 2015 (2). Although radical surgery is currently the most effective treatment for $\mathrm{PC}$, the incidence of early tumor recurrence after surgery is high, which leads to a reduction in long-term survival. Therefore, the identification of specific biomarkers to predict early postoperative recurrence is of great value in improving the prognosis of $\mathrm{PC}$.

Heat shock-related $70-\mathrm{kDa}$ proteins (HSP70s) are a class of hot shock proteins (HSPs) with a molecular weight of about $70-\mathrm{kD}$, they usually play the role of molecular chaperones, and also have antioxidant, antiapoptotic and immunomodulatory effects (3). HSPA2, also named HSP70-2, is one of the significant members of the HSP70s family, originally found in male germ cells and described as a testicle-specific protein that is essential in spermatogenesis (4-6). The HSPA2 gene is located on chromosome 14 (14q24.1), and its abnormal expression in the testis causes meiosis to be unsuccessful, leading to male sterility $(4,7)$. Previous studies have reported that HSPA2 was overexpressed in several human solid neoplasms, including cervical malignancies (8), bladder urothelial cancer (9), esophageal squamous cell cancer (ESCC) (10), non-small cell lung cancer (NSCLC) (11), hepatocellular cancer (HCC) $(12,13)$, colorectal cancer (14), breast cancer $(15-17)$, and PC $(18,19)$. These reports show that HSPA2 overexpression acts as a vital function in the genesis and progress of carcinoma.

It has been demonstrated that the upregulation of HSPA2 in cancerous tissues is tightly interrelated with the shortened postoperative survival of PC $(18,19)$. However, the relationship between HSPA2 and early postoperative recurrence of $\mathrm{PC}$ remains unclear. Therefore, this study assessed HSPA2 expression in PC tissues by immunohistochemistry and analyzed its correlation with early postoperative recurrence of $\mathrm{PC}$.

We present the following article in accordance with the STARD reporting checklist (available at https://dx.doi. org/10.21037/gs-21-262).

\section{Methods}

\section{Tissue samples and clinicopathologic data}

The cancerous and adjacent paracancerous specimens were gathered from 85 PC cases that achieved radical excision between January 2008 and December 2011. All patients were confirmed as PC by pathology and had not received presurgical anticancer treatment, including chemotherapy, radiotherapy, chemoradiotherapy, biologically targeted treatment, and/or immunotherapy. All patients included in the study had the entire tumor removed without residual tumor cells (R0 resection). The samples were immersed in formaldehyde solution within 30 minutes after excision for fixation and stored in the Biobank after embedding in paraffine. Paracancerous tissues refer to normal pancreas tissues which situate $2 \mathrm{~cm}$ or more away from the edge of the confirmed neoplasms. The study was conducted in accordance with the Declaration of Helsinki (as revised in 2013). The study was approved by the ethics board of Renmin Hospital of Wuhan University (NO. WDRY2019-K070) and individual consent for this retrospective analysis was waived.

The clinicopathologic parameters of PC were collected retrospectively from the medical records (Electronic Medical Record), including age, sex, tumor site, tumor size, presurgical serum carbohydrate antigen 19-9 (CA19-9) measurement values, tumor differentiation grade, lymph node metastasis (LNM) pattern, peripheral nerve infiltration (PNI) pattern, tumor staging status and early postsurgical relapse state. The tumor differentiation was histologically categorized into moderate-poor and well differentiation. The tumor staging was determined with the 8th edition of the AJCC TNM staging system (20). All cases were followed up continuously after radical resection. Early postsurgical recurrence of PC was designated as the neoplasm recurred within one year following radical surgery (21). Postoperative recurrence includes local recurrence (e.g., residual pancreas, anastomosis, etc.) and distant metastases (e.g., liver, peritoneum, abdominal lymph nodes, lungs, bone, etc.).

\section{Immunobistochemical analysis}

The expression of HSPA2 in clinical samples was detected by immunohistochemical staining, as formerly reported (19). The paraffin-embedded specimens were made into 4-micron contiguous tissues and attached to glass slides. 
Next, the sections with tissues were placed in a heater at $60{ }^{\circ} \mathrm{C}$ for $60 \mathrm{~min}$ to melt the paraffin. The tissue sections were then treated with xylene to dewax and with different concentrations of alcohol to hydrate. After that, the tissue slices were immerged into sodium citrate-hydrochloric acid buffer solution $(0.01 \mathrm{~mol} / \mathrm{L}, \mathrm{pH} 6.0)$ and heated by microwave at $80{ }^{\circ} \mathrm{C}$ for $20 \mathrm{~min}$ to achieve antigenic retrieval. After quenching the endogenetic peroxidase function with $3 \%$ deionized $\mathrm{H}_{2} \mathrm{O}_{2}$ solution, the tissue sections were incubated with monoclonal rabbit anti-human HSPA2 primary antibody (Abcam Company, Cambridge, England, UK) at $4{ }^{\circ} \mathrm{C}$ overnight. After rewarming, the sections were cleaned in phosphate-buffered saline (PBS) and then incubated with goat anti-rabbit HRP-labeled IgG secondary antibody (Zhongshan Golden Bridge Biotechnology Co., Ltd., Beijing, China) at room temperature for 30 minutes. Finally, the sections were sequentially subjected to chromogenic reaction, counterstaining, dehydration, cleaning, and mounting. The section was not incubated with the HSPA2 antibody served as a negative control.

Immunostaining results were scored independently and semi-quantitatively by two researchers participating in the experiment based on the percent of staining cells and the degree of color, as mentioned formerly (19). The scoring range for the percent of staining cells was as follows: 0 marks means nonstained cells, 1 mark means $1-10 \%$ staining cells, 2 marks means $10-30 \%$ staining cells, and 3 marks means $>30 \%$ staining cells (19). The scoring range of immunostaining degree was recorded as: 0 for colorless, 1 for weak coloring, 2 for middle coloring, 3 for strong coloring (19). The final total score for each section was the staining percentage score multiplied by the staining degree score. For statistical purpose, a total point of less than 3 was designated as low expression and no less than 3 as high expression. The difference in scores was resolved through a joint evaluation.

\section{Statistical analysis}

The statistical analyses were conducted with SPSS version 17.0 (SPSS, Inc., Chicago, IL, USA), Microsoft Office Excel 2007 (Microsoft, Redmond, WA, USA), and R software version 4.0.3. Categorical variables were expressed as the number of cases and percentages, and the statistic analysis between the groups was estimated by Pearson's chisquare test or Fisher's exact test. Logistic regression analysis was applied to determine risk variables related to early postoperative recurrence. Variables that showed statistically significant in univariate logistic regression analyses were incorporated into a multivariate logistic regression model to identify independent predictors of early postoperative relapse. The Hosmer-Lemeshow test was used to estimate the goodness of fit of the multivariate logistic regression model. Receiver operating characteristic (ROC) curve analysis was applied to assess the predictive power of risk factors for early relapse of PC after surgery. The clinical utility evaluation of risk factors was performed by decision curve analysis (DCA). A two-tailed P value of less than 0.05 was suggested to be statistically significant.

\section{Results}

\section{Clinicopathologic features of PC patients}

A total of 85 PC patients were included in this study. The average age of these PC patients was $56.9 \pm 6.5$ years (ranging from 44 to 68 years), including 45 men and 40 women. There were 41 patients with tumor size less than $20 \mathrm{~mm}, 44$ patients with tumor size greater than or equal to $20 \mathrm{~mm}, 71$ patients with pancreatic head cancer, 14 patients with pancreatic body/tail carcinoma, 38 patients with serum CA19-9 $\leq 37 \mathrm{U} / \mathrm{mL}, 47$ patients with $>37 \mathrm{U} / \mathrm{mL}$. Postoperative pathological examination confirmed that 29 patients with moderate/poor tumor differentiation, 56 patients with well tumor differentiation, 26 patients with non-metastatic lymph nodes, 59 patients with metastatic lymph nodes, 49 patients without PNI, 36 patients with PNI, 23 patients with stage I, and 62 patients with stage II. Thirty-five cases without early postsurgical recurrence and 50 cases with early postsurgical recurrence. Among the patients with early postoperative relapse, 14 presented with local recurrence, 29 with liver metastases, 3 with peritoneal metastases, 3 with abdominal lymph node metastases, and 1 with pulmonary metastases. A summary of clinicopathologic parameters is shown in Table 1 .

\section{Expression of HSPA2 in PC tissues}

Immunostaining indicated that HSPA2 was largely distributed in the cytoplasmic space, as shown in Figure $1 \mathrm{~A}$. Of the 85 PC specimens, overexpressed HSPA2 was observed in $70.6 \%(60 / 85)$. However, only $25.9 \%(22 / 85)$ of HSPA2 overexpression was found in the matched nontumor specimens. Statistical analysis revealed that HSPA2 expression was signally increased in $\mathrm{PC}$ specimens relative to surrounding para-carcinoma specimens $(\mathrm{P}=0.003$; Figure $1 \mathrm{~B}$ 
Table 1 Clinicopathologic data of 85 PC patients

\begin{tabular}{|c|c|c|c|}
\hline Clinicopathologic data & Group & Number & $\%$ \\
\hline Age (years) & $\geq 60$ & 34 & 40.0 \\
\hline \multirow[t]{2}{*}{ Sex } & Male & 45 & 52.9 \\
\hline & Female & 40 & 47.1 \\
\hline Tumor size (mm) & $\geq 20$ & 44 & 51.8 \\
\hline \multirow[t]{2}{*}{ Tumor site } & Head & 71 & 83.5 \\
\hline & Body/tail & 14 & 16.5 \\
\hline Serum CA19-9 (U/mL) & $\leq 37$ & 38 & 44.7 \\
\hline Tumor differentiation & Moderate/poor & 56 & 65.9 \\
\hline \multirow[t]{2}{*}{ LNM } & Absent & 26 & 30.6 \\
\hline & Present & 59 & 69.4 \\
\hline \multirow[t]{2}{*}{$\mathrm{PNI}$} & Absent & 49 & 57.6 \\
\hline & Present & 36 & 42.4 \\
\hline \multirow[t]{2}{*}{ Tumor stage } & I & 23 & 27.1 \\
\hline & $\|$ & 62 & 72.9 \\
\hline Early postoperative recurrence & No & 35 & 41.2 \\
\hline
\end{tabular}

PC, pancreatic cancer; CA19-9, carbohydrate antigen19-9; LNM, lymph node metastasis; PNI, peripheral nerves infiltration. Neoplasm recurrence within 12 months after surgery was defined as early postoperative relapse.

and Table 2). HSPA2 expression was observably higher in tumors with early postsurgical relapse compared with those without early postsurgical relapse $(\mathrm{P}<0.001$; Figure $1 C$ and Table 3). Moreover, HSPA2 expression was markedly linked to malignant clinicopathologic features such as preoperative serum CA19-9, tumour differentiation, LNM, PNI and tumour stage (Table 4).

\section{Logistic regression analysis of risk variables potentially associated with early postsurgical relapse of PC}

To ascertain the variates that pre-estimate the early postsurgical recurrence of $\mathrm{PC}$, we carried out univariate and multivariate analyses with logistic regression models. Univariate logistic regression analysis revealed that early neoplasm relapse was observably correlated with high serum CA19-9 levels before surgery $(\mathrm{P}=0.019)$, moderate/ poor tumor differentiation $(\mathrm{P}=0.001)$, the presence of $\mathrm{LNM}$ $(\mathrm{P}<0.001)$, the presence of $\mathrm{PNI}(\mathrm{P}=0.011)$, high tumor staging $(\mathrm{P}<0.001)$, and overexpressed HSPA2 $(\mathrm{P}<0.001)$ in $\mathrm{PC}$ patients after resection (Figure $2 A$ and Table $\mathrm{S} 1$ ).

In the multivariate logistic regression model, upregulated HSPA2 proved to be an independent hazard variate for early recidivation of $\mathrm{PC}$ following surgery [odds ratio $(\mathrm{OR})=6.601 ; 95 \%$ confidence interval $(\mathrm{CI}): 1.635-26.652$; $\mathrm{P}=0.008]$. The high tumor stage was also shown to be an independent variable for predicting early relapse of $\mathrm{PC}$ after the operation ( $\mathrm{OR}=8.049 ; 95 \% \mathrm{CI}: 1.783-36.334 ; \mathrm{P}=0.007)$ (Figure 2B and Table S1). The Hosmer-Lemeshow test showed a $\mathrm{P}$ value of 0.642 (greater than 0.05 ), indicating that this multivariate logistic regression model had good goodness of fit. 
A

Pancreatic cancer tissue

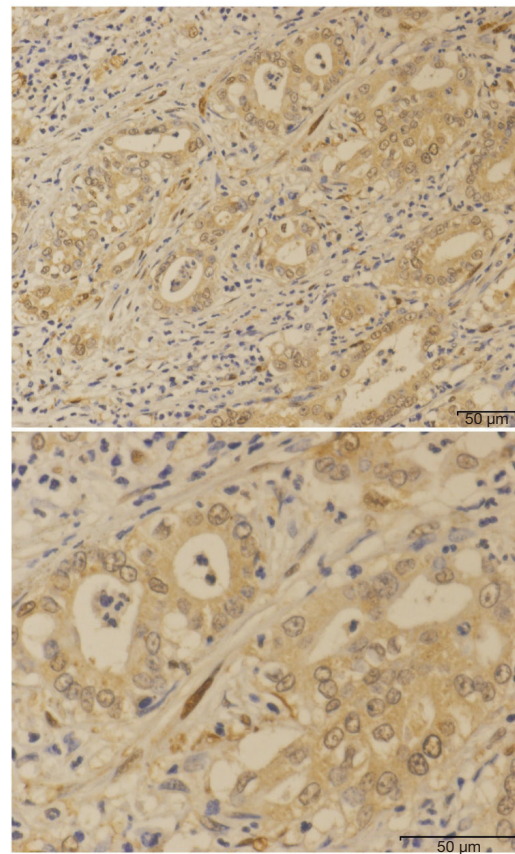

Paracancerous tissue

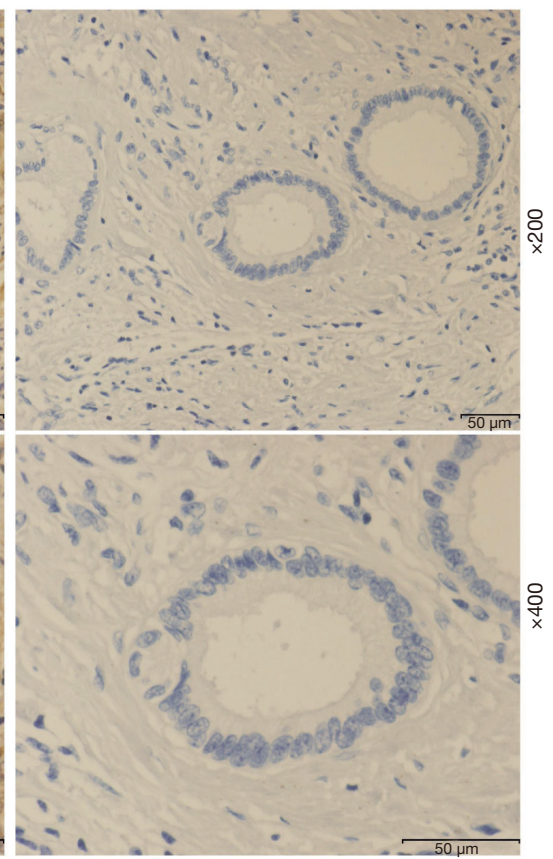

B

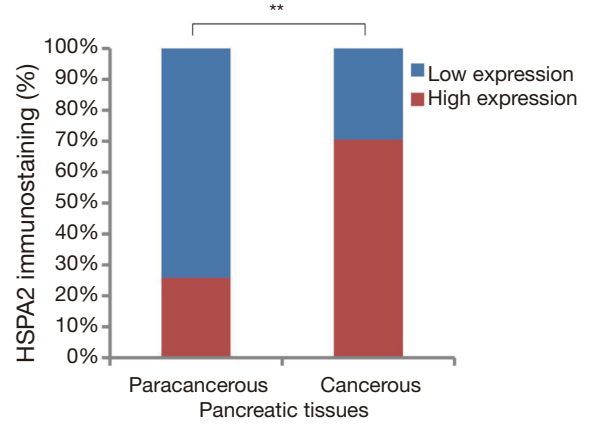

C

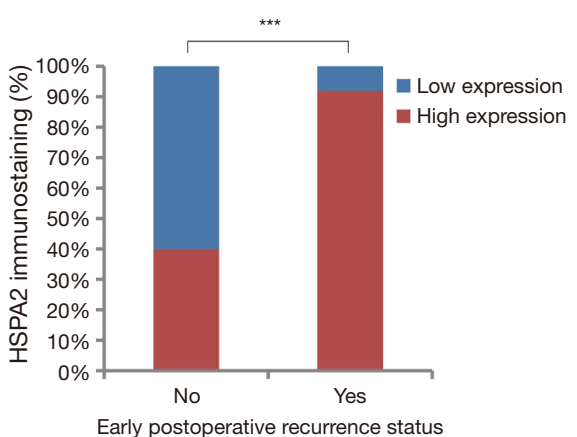

Figure 1 Expression of HSPA2 in PC tissues. (A) Representative photographs of HSPA2 immunostaining in cancer and paracancerous tissues were taken at different magnifications (top panel $\times 200$, bottom panel $\times 400$ ). High expression of HSPA2 was observed in cancer tissues, mainly in the cytoplasm, while the expression of HSPA2 was low in paracancerous tissues. (B) High expression rate of HSPA2 in cancer tissues was significantly higher than that in adjacent paracancerous tissues. (C) High expression rate of HSPA2 in tumors with early postoperative relapse was significantly higher than that of tumors without early postoperative relapse. HSPA2, heat shock protein A2; PC, pancreatic cancer. ${ }^{* *} \mathrm{P}<0.01,{ }^{* * *} \mathrm{P}<0.001$.

Table 2 Expression of HSPA2 in 85 pairs of cancerous and adjacent paracancerous clinical specimens based on immunohistochemistry

\begin{tabular}{|c|c|c|c|c|}
\hline \multirow{2}{*}{ Pancreatic tissues } & \multirow{2}{*}{ Number } & \multicolumn{2}{|c|}{ HSPA2 expression } & \multirow{2}{*}{$P$ value } \\
\hline & & Low & High & \\
\hline Cancerous & 85 & 25 & 60 & 0.003 \\
\hline Paracancerous & 85 & 63 & 22 & \\
\hline
\end{tabular}

The semi-quantitative score value 3 of immunohistochemistry was used as the cut-off value for high and low expression of HSPA2. Data were analyzed by chi-squared test. $\mathrm{P}<0.05$ indicates statistical significance of differences. HSPA2, heat shock protein A2.

Table 3 Differential expression of HSPA2 between patients with early postoperative recurrence and patients without early postoperative recurrence $(n=85)$

\begin{tabular}{|c|c|c|c|c|}
\hline Early postoperative recurrence status & Number & \multicolumn{2}{|c|}{ HSPA2 expression } & $P$ value \\
\hline No & 35 & 21 & 14 & $<0.001$ \\
\hline Yes & 50 & 4 & 46 & \\
\hline
\end{tabular}

The semi-quantitative score value 3 of immunohistochemistry was used as the cut-off value for high and low expression of HSPA2. Data were analyzed by chi-squared test. $\mathrm{P}<0.05$ indicates statistical significance of differences. HSPA2, heat shock protein A2. 
Table 4 Correlations between HSPA2 expression and clinicopathologic characteristics of PC (n=85)

\begin{tabular}{|c|c|c|c|c|c|}
\hline Clinicopathologic characteristics & Group & Number & \multicolumn{2}{|c|}{ HSPA2 expression } & $P$ value \\
\hline \multirow[t]{2}{*}{ Age (years) } & $<60$ & 51 & 14 & 37 & 0.627 \\
\hline & $\geq 60$ & 34 & 11 & 23 & \\
\hline Sex & Male & 45 & 14 & 31 & 0.715 \\
\hline \multirow[t]{2}{*}{ Tumor size (mm) } & $<20$ & 41 & 13 & 28 & 0.654 \\
\hline & $\geq 20$ & 44 & 12 & 32 & \\
\hline \multirow[t]{2}{*}{ Tumor site } & Head & 71 & 23 & 48 & 0.174 \\
\hline & Body/tail & 14 & 2 & 12 & \\
\hline \multirow[t]{2}{*}{ Tumor differentiation } & Well & 29 & 19 & 10 & $<0.001$ \\
\hline & Moderate/poor & 56 & 6 & 50 & \\
\hline \multirow[t]{2}{*}{ LNM } & Absent & 26 & 18 & 8 & $<0.001$ \\
\hline & Present & 59 & 7 & 52 & \\
\hline \multirow[t]{2}{*}{$\mathrm{PNI}$} & Absent & 49 & 21 & 28 & 0.002 \\
\hline & Present & 36 & 4 & 32 & \\
\hline \multirow[t]{2}{*}{ Tumor stage } & I & 23 & 18 & 5 & $<0.001$ \\
\hline & II & 62 & 7 & 55 & \\
\hline
\end{tabular}

The semi-quantitative score value 3 of immunohistochemistry was used as the cut-off value for high and low expression of HSPA2. Data were analyzed by chi-squared test. $\mathrm{P}<0.05$ indicates statistical significance of differences. HSPA2, heat shock protein A2; PC, pancreatic cancer; CA19-9, carbohydrate antigen19-9; LNM, lymph node metastasis; PNI, peripheral nerves infiltration.

\section{Predictive performance and potential application value of HSPA2 for early postsurgical relapse}

ROC curves were constructed to assess the reliability of these risk factors for predicting early relapse of PC following surgery. The area under curve (AUC) was 0.760 (95\% CI: $0.649-0.871$ ) for HSPA2, 0.630 (95\% CI: $0.509-0.751)$ for serum CA19-9, 0.671 (95\% CI: $0.552-0.791)$ for tumor differentiation, 0.750 (95\% CI: 0.638-0.862) for LNM, 0.641 (95\% CI: $0.522-0.760$ ) for PNI, 0.756 (95\% CI: 0.643-0.868) for tumor stage. The combination of HSPA2 and the above-mentioned malignant clinicopathologic parameters improved the specificity and sensitivity over that of HSPA2 or these clinicopathologic parameters alone for predicting early postsurgical relapse of PC, with an AUC of 0.843 (95\% CI: 0.753-0.932) (Figure 2C).
DCA was employed to quantify the probability of net benefits of risk factors to further assess their predictive performance and potential clinical application value for early recurrence of PC after surgery. The results demonstrated that HSPA2 expression had a larger net benefit than other risk variables. The combination of all risk variables presented the highest net benefit relative to individual risk variables, indicating that HSPA2 expression combined with other malignant clinicopathologic characteristics had a great advantage in predicting the possibility of early postoperative relapse (Figure 2D).

\section{Discussion}

HSPA2 expression has been confirmed to be elevated in a variety of human solid malignancies, including PC (819). Although overexpression of HSPA2 in PC tissues has 
A

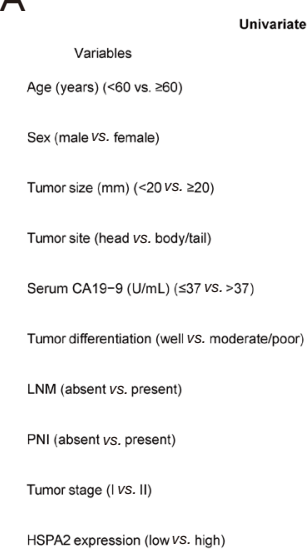

C

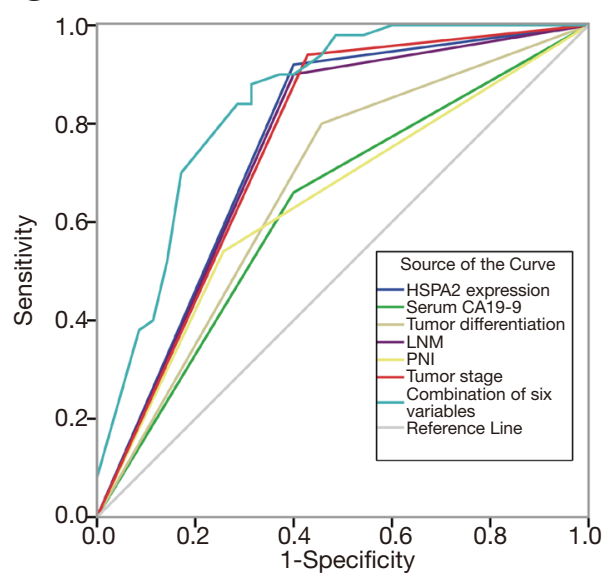

B

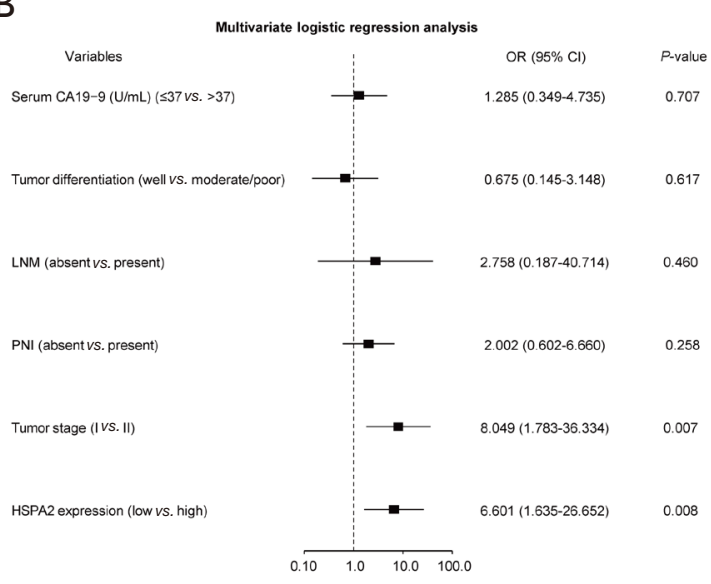

D

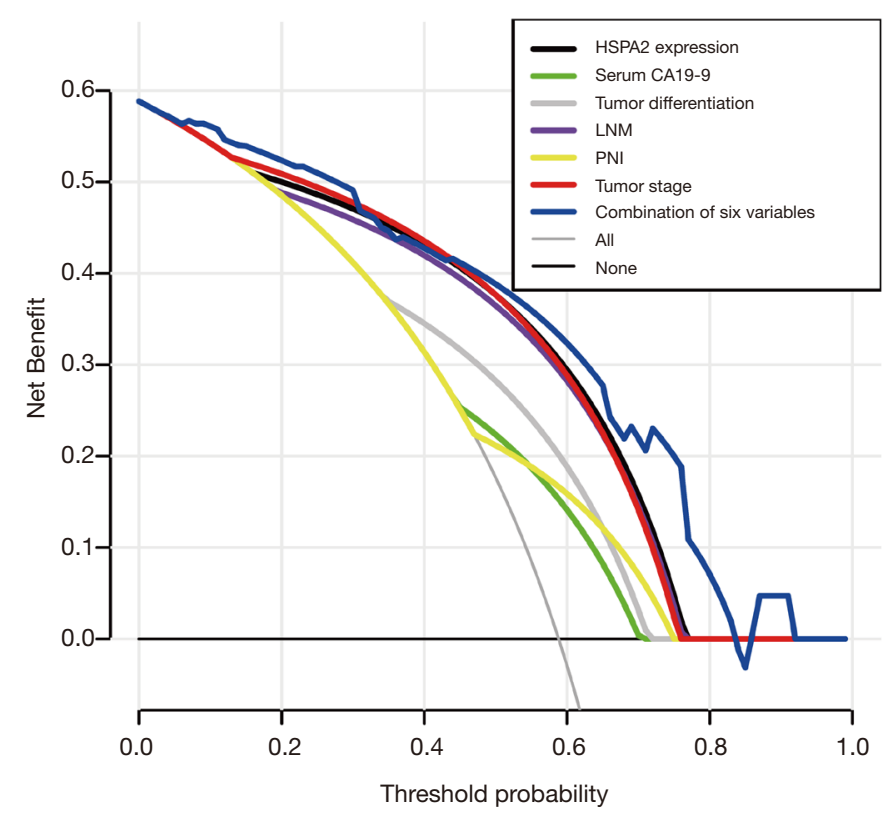

Figure 2 Prediction of risk variables for early postsurgical recurrence of PC. (A,B) Forest plots for univariate and multivariate logistic regression analysis. High HSPA2 expression, high preoperative serum CA19-9 levels, moderate/poor tumor differentiation, the presence of LNM, the presence of PNI, and late tumor stage were risk factors for early postoperative recurrence. High HSPA2 expression and late tumor stage were independent risk factors. (C) ROC curves analysis of risk variables for predicting the early postsurgical relapse. The AUC for HSPA2 expression, serum CA19-9, tumor differentiation, LNM, PNI, tumor stage and the combination of these variables (combination of six variables) were $0.760,0.630,0.671,0.750,0.641,0.756$, and 0.843 , respectively. (D) DCA of risk variables for predicting the early postsurgical relapse. The $\mathrm{Y}$-axis and $\mathrm{X}$-axis represent the net benefit and the threshold probability, respectively. The two curves of "All" and "None" mean respectively assuming that all patients have an early postoperative relapse and that no patients have an early postsurgical recurrence. HSPA2 expression, serum CA19-9, tumor differentiation, LNM, PNI, tumor stage, and the combination of these variables (combination of six variables) had more net benefits than the two extreme cases (All and None) across the area of appropriate threshold probabilities. HSPA2 expression was superior to the other five clinicopathologic features in predicting early postoperative relapse. The combination of six variables was better than HSPA2 expression or clinicopathologic parameters alone. Net benefit = (number of true positives/overall number of cases) - (number of false positives/overall number of cases) $\times$ [threshold probability/(1-threshold probability)]. PC, pancreatic cancer; CA19-9, carbohydrate antigen 19-9; LNM, lymph node metastasis; PNI, peripheral nerves infiltration; HSPA2, heat shock protein A2; OR, odds ratio; CI, confidence interval; ROC, receiver operating characteristic; AUC, area under the curve; DCA, decision curve analysis. $\mathrm{P}<0.05$ indicates statistically significant. 
been confirmed to be strongly correlative with shortened survival time, the relationship between HSPA2 and early postsurgical recurrence of PC remains unclear. Therefore, this study explored the associativity between HSPA2 expression and early reappearance of PC after surgery. The findings showed that HSPA2 expression in PC specimens was markedly enhanced relative to the nontumor specimens; which was in accord with the former results $(18,19)$. Elevated HSPA2 expression served as an independent risk indicator for early postsurgical relapse of PC. ROC curve analysis showed that HSPA2 expression combined with other malignant clinicopathologic parameters had a larger AUC value. DCA indicated that HSPA2 expression combined with other malignant clinicopathologic features had a higher net benefit than HSPA2 or clinicopathologic parameters alone.

There is mounting evidence that HSPA2 expression is closely associative with the aggressive progression of tumors. In cervical cancer, bladder urothelial cancer, colorectal cancer, and breast cancer, the depletion of HSPA2 expression observably reduced the proliferation, invasion and migration abilities of carcinoma cells, initiated cell apoptosis, and restrained the increase in the volume of transplanted tumors $(8,9,14-16,22)$. In kidney cancer, ablated HSPA2 in tumor cells caused the attenuation of vitality, colony formation and movement (23). In ovarian cancer cells, HSPA2 silencing induced by shRNA resulted in the decrease of cell proliferation, viability, colony formation and cell motility capacity, and the cell cycle was arrested in the G0-G1 phase (24). In lung adenocarcinoma, siRNA-mediated downregulation of HSPA2 caused the reduction of cell activity and cell cycle arrest in the G1-S stage (25). Additionally, some studies have shown that HSPA2 overexpression was prominently associated with malignant clinicopathologic characteristics of several human malignant neoplasms, including ESCC (10), NSCLC (11), HCC (12), breast cancer (17) and PC $(18,19)$. Our data also confirmed that high expression of HSPA2 in neoplastic tissues was strongly linked to malignant clinicopathologic features such as poor tumor differentiation, tumor nerve infiltration, tumor lymphatic metastasis, and more advanced tumor staging. The current findings, together with previous data imply an important contribution of HSPA2 in the malignant progression of cancer.

Correlations between HSPA2 overexpression and clinical outcomes in several malignant tumors have been reported. For instance, Zhang et al. (10) reported that increased HSPA2 expression was apparently associated with a declining survival rate and independently forecasted the adverse outcome in ESCC individuals. Scieglinska et al. (11) demonstrated that HSPA2 upregulation was markedly relevant to shortened overall survival time in the NSCLC population with stages I and II. Fu et al. (12) suggested that HCC with upregulated HSPA2 presented a less overall survival time than those with downregulated HSPA2, and HSPA2 overexpression could be used as an independent adverse variable for prognosis prediction. Yang et al. (17) showed that overexpression of HSPA2 was clearly connected with reduced survival time of breast cancer patients. Moreover, previous studies have also shown that elevatory HSPA2 expression was significantly linked to the shortened recurrence-free survival and overall survival of PC cases $(18,19)$. Nevertheless, the relationship between HSPA2 and early recurrence of PC after surgery is still unexplored. Although serum CA19-9 has been reported to be associated with early relapse of $\mathrm{PC}$ after resection, it still lacks sensitivity and specificity $(26,27)$. Therefore, it would be of great benefit to seek additional tumor markers, such as HSPA2, as predictors of early postsurgical recurrence of PC. In the present work, we found that upregulated HSPA2 was evidently correlated with early postoperative relapse of PC and was an independent hazard indicator of early postoperative recurrence. Furthermore, HSPA2 expression combined with other malignant clinicopathologic characteristics significantly improved the reliability for the prediction of early postsurgical recurrence, with a great potential application value. These findings together suggest that HSPA2 overexpression is tightly connected with the unfavorable outcome of human malignant tumors, which further reveals the important role of its gene in the progress and recurrence of malignancies.

We have to acknowledge the deficiencies of this research. First, the establishment of logistic regression models that predict the risk factors for early postoperative relapse of PC was based on a retrospective analysis of a single institution with a relatively small sample capacity, so there is ineluctably a selection bias. Second, we did not investigate the effect of neoadjuvant therapy on HSPA2 expression, and further studies are needed to reveal the clinical relevance of HSPA2 to neoadjuvant therapy. Third, the current study did not determine the effect and mechanism of HSPA2 expression on the biological behavior of PC cells. Finally, this study did not explore the influence of HSPA2 on the biological behaviors of transplanted tumours in animals. Consequently, it is necessary to collect more clinical data, apply more experimental techniques, and design more 
rigorous research protocols to further validate the current findings and reveal the biological role of HSPA2 in PC.

\section{Conclusions}

In conclusion, the current results indicate that high expression of intratumoral HSPA2 is strongly linked to early relapse of PC after resection and acts as an independent predictor of early postsurgical recurrence. The combination of HSPA2 expression and other malignant clinicopathologic parameters shows better performance and greater potential clinical value in predicting early postsurgical recurrence of PC. These data reveal that HSPA2 has a significant part in cancer progress and is a potential tumor marker for predicting early postoperative recurrence of PC.

\section{Acknowledgments}

The authors thank the Anhui Provincial Hospital and the Anhui Province Key Laboratory of Hepatopancreatobiliary Surgery for providing experimental materials and equipment. We would like to thank Dr. A-Kao Zhu for his help in polishing our paper.

Funding: This study was supported by the Zhejiang Provincial Science and Technology project for Medicine and Health of China (No. 2018KY579), Zhejiang Provincial Natural Science Foundation of China (No. LGF19H290004), Science and Technology Development Foundation of Nanjing Medical University (No. 2017NJMU084), and National Natural Science Foundation of China (No. 81272740).

\section{Footnote}

Reporting Checklist: The authors have completed the STARD reporting checklist. Available at https://dx.doi. org/10.21037/gs-21-262

Data Sharing Statement: Available at https://dx.doi. org/10.21037/gs-21-262

Conflicts of Interest: All authors have completed the ICMJE uniform disclosure form (available at https://dx.doi. org/10.21037/gs-21-262). The authors have no conflicts of interest to declare.

Ethical Statement: The authors are accountable for all aspects of the work in ensuring that questions related to the accuracy or integrity of any part of the work are appropriately investigated and resolved. The study was conducted in accordance with the Declaration of Helsinki (as revised in 2013). The study was approved by the ethics board of Renmin Hospital of Wuhan University (NO. WDRY2019-K070) and individual consent for this retrospective analysis was waived.

Open Access Statement: This is an Open Access article distributed in accordance with the Creative Commons Attribution-NonCommercial-NoDerivs 4.0 International License (CC BY-NC-ND 4.0), which permits the noncommercial replication and distribution of the article with the strict proviso that no changes or edits are made and the original work is properly cited (including links to both the formal publication through the relevant DOI and the license). See: https://creativecommons.org/licenses/by-nc-nd/4.0/.

\section{References}

1. Bray F, Ferlay J, Soerjomataram I, et al. Global cancer statistics 2018: GLOBOCAN estimates of incidence and mortality worldwide for 36 cancers in 185 countries. CA Cancer J Clin 2018;68:394-424.

2. Siegel RL, Miller KD, Jemal A. Cancer statistics, 2020. CA Cancer J Clin 2020;70:7-30.

3. Glover JR, Lindquist S. Hsp104, Hsp70, and Hsp40: a novel chaperone system that rescues previously aggregated proteins. Cell 1998;94:73-82.

4. Bonnycastle LL, Yu CE, Hunt CR, et al. Cloning, sequencing, and mapping of the human chromosome 14 heat shock protein gene (HSPA2). Genomics 1994;23:85-93.

5. Dix DJ, Allen JW, Collins BW, et al. HSP70-2 is required for desynapsis of synaptonemal complexes during meiotic prophase in juvenile and adult mouse spermatocytes. Development 1997;124:4595-603.

6. Son WY, Han CT, Hwang SH, et al. Repression of hspA2 messenger RNA in human testes with abnormal spermatogenesis. Fertil Steril 2000;73:1138-44.

7. Dix DJ, Allen JW, Collins BW, et al. Targeted gene disruption of Hsp70-2 results in failed meiosis, germ cell apoptosis, and male infertility. Proc Natl Acad Sci U S A 1996;93:3264-8.

8. Garg M, Kanojia D, Saini S, et al. Germ cell-specific heat shock protein 70-2 is expressed in cervical carcinoma and is involved in the growth, migration, and invasion of cervical cells. Cancer 2010;116:3785-96.

9. Garg M, Kanojia D, Seth A, et al. Heat-shock protein 70-2 
(HSP70-2) expression in bladder urothelial carcinoma is associated with tumour progression and promotes migration and invasion. Eur J Cancer 2010;46:207-15.

10. Zhang H, Chen W, Duan CJ, et al. Overexpression of HSPA2 is correlated with poor prognosis in esophageal squamous cell carcinoma. World J Surg Oncol 2013;11:141.

11. Scieglinska D, Gogler-Piglowska A, Butkiewicz D, et al. HSPA2 is expressed in human tumors and correlates with clinical features in non-small cell lung carcinoma patients. Anticancer Res 2014;34:2833-40.

12. Fu Y, Zhao H, Li XS, et al. Expression of HSPA2 in human hepatocellular carcinoma and its clinical significance. Tumour Biol 2014;35:11283-7.

13. Yang Z, Zhuang L, Szatmary P, et al. Upregulation of heat shock proteins (HSPA12A, HSP90B1, HSPA4, HSPA5 and HSPA6) in tumour tissues is associated with poor outcomes from HBV-related early-stage hepatocellular carcinoma. Int J Med Sci 2015;12:256-63.

14. Jagadish N, Parashar D, Gupta N, et al. Heat shock protein 70-2 (HSP70-2) is a novel therapeutic target for colorectal cancer and is associated with tumor growth. BMC Cancer 2016;16:561.

15. Rohde M, Daugaard M, Jensen MH, et al. Members of the heat-shock protein 70 family promote cancer cell growth by distinct mechanisms. Genes Dev 2005;19:570-82.

16. Jagadish N, Agarwal S, Gupta N, et al. Heat shock protein 70-2 (HSP70-2) overexpression in breast cancer. J Exp Clin Cancer Res 2016;35:150.

17. Yang YL, Zhang Y, Li DD, et al. RNF144A functions as a tumor suppressor in breast cancer through ubiquitin ligase activity-dependent regulation of stability and oncogenic functions of HSPA2. Cell Death Differ 2020;27:1105-18.

18. Zhang H, Gao H, Liu C, et al. Expression and clinical signifificance of HSPA2 in pancreatic ductal adenocarcinoma. Diagn Pathol 2015;10:13.

Cite this article as: Zhai LL, Qiao PP, Sun YS, Tang ZG, Ju TF. Upregulated HSPA2 predicts early relapse of pancreatic cancer after surgery. Gland Surg 2021;10(7):2140-2149. doi: 10.21037 /gs-21-262
19. Zhai LL, Xie Q, Zhou CH, et al. Overexpressed HSPA2 correlates with tumor angiogenesis and unfavorable prognosis in pancreatic carcinoma. Pancreatology 2017; 17:457-63.

20. Amin MB, Edge S, Greene F, et al. American Joint Committee on Cancer. AJCC cancer staging manual. 8th ed. New York, NY: Springer 2017.

21. Yamamoto Y, Ikoma H, Morimura R, et al. Optimal duration of the early and late recurrence of pancreatic cancer after pancreatectomy based on the difference in the prognosis. Pancreatology 2014;14: 524-9.

22. Daugaard M, Kirkegaard-Sørensen T, Ostenfeld MS, et al. Lens epithelium-derived growth factor is an Hsp70-2 regulated guardian of lysosomal stability in human cancer. Cancer Res 2007;67:2559-67.

23. Singh S, Suri A. Targeting the testis-specific heatshock protein 70-2 (HSP70-2) reduces cellular growth, migration, and invasion in renal cell carcinoma cells. Tumour Biol 2014;35:12695-706.

24. Gupta N, Jagadish N, Surolia A, et al. Heat shock protein 70-2 (HSP70-2) a novel cancer testis antigen that promotes growth of ovarian cancer. Am J Cancer Res 2017;7:1252-69.

25. Cao L, Yuan X, Bao F, et al. Downregulation of HSPA2 inhibits proliferation via ERK1/2 pathway and endoplasmic reticular stress in lung adenocarcinoma. Ann Transl Med 2019;7:540.

26. Daamen LA, Groot VP, Heerkens HD, et al. Systematic review on the role of serum tumor markers in the detection of recurrent pancreatic cancer. HPB (Oxford) 2018;20:297-304.

27. van Manen L, Groen JV, Putter H, et al. StageSpecific Value of Carbohydrate Antigen 19-9 and Carcinoembryonic Antigen Serum Levels on Survival and Recurrence in Pancreatic Cancer: A Single Center Study and Meta-Analysis. Cancers (Basel) 2020;12:2970. 


\section{Supplementary}

Table S1 Logistic regression analysis of factors associated with early postoperative recurrence of PC

\begin{tabular}{|c|c|c|c|c|}
\hline Variables & \multicolumn{2}{|c|}{ Univariate analysis } & \multicolumn{2}{|c|}{ Multivariate analysis } \\
\hline Age (years) $(<60$ vs. $\geq 60)$ & $1.225(0.505-2.973)$ & 0.653 & & \\
\hline Sex (male vs. female) & $1.625(0.678-3.897)$ & 0.277 & & \\
\hline Tumor size (mm) (<20 vs. $\geq 20)$ & $1.511(0.634-3.602)$ & 0.351 & & \\
\hline Serum CA19-9 (U/mL) ( $\leq 37$ vs. $>37)$ & $2.912(1.191-7.121)$ & 0.019 & $1.285(0.349-4.735)$ & 0.707 \\
\hline Tumor differentiation (well vs. moderate/poor) & $4.750(1.818-12.411)$ & 0.001 & $0.675(0.145-3.148)$ & 0.617 \\
\hline LNM (absent vs. present) & $13.500(4.296-42.422)$ & $<0.001$ & $2.758(0.187-40.714)$ & 0.460 \\
\hline PNI (absent vs. present) & $3.391(1.325-8.683)$ & 0.011 & $2.002(0.602-6.660)$ & 0.258 \\
\hline
\end{tabular}

PC, pancreatic cancer; CA19-9, carbohydrate antigen19-9; LNM, lymph node metastasis; PNI, peripheral nerves infiltration; HSPA2, heat shock protein A2; OR, odds ratio; Cl, confidence interval. The HSPA2 aggregate score $<3$ was defined as low expression, and $\geq 3$ as high expression. $\mathrm{P}<0.05$ indicates statistically significant. 